\title{
LA ADAPTACIÓN Y VALIDACIÓN AL ESPAÑOL DE UNA BATERÍA DE 27 ESCALAS (MPSI) PARA MEDIR PROBLEMAS PSICOSOCIALES CON UNA MUESTRA PUERTORRIQUEÑA
}

\author{
Jaime Alvelo Burgos ${ }^{1}$ \\ Luz Enid Del Valle ${ }^{2}$
}

\section{Introducción}

\begin{abstract}
O
olón Castillo (2000) señala que tanto las metodologías cualita 1 tivas como las cuantitativas de medición son herramientas valiosas para la evaluación de logros de los objetivos trazados para la intervención. En una época en donde cada día se hace más patente la necesidad el rendir cuentas a la sociedad por nuestras ejecutorias profesionales es un reto obtener herramientas de medición válidas y confiables. Los autores de este artículo al encontrarse con la escasez de escalas estandarizadas para evaluar su práctica se dieron a la tarea de adaptar a Puerto Rico El Inventario para el Cernimiento de Múltiples Problemas ó MPSI por sus siglas en inglés de Hudson (1997). Se escogió este instrumento de medición ya que uno de los autores asistió a un adiestramiento sobre la administración del MPSI dado por el autor del mismo, entendiendo entonces su utilidad para la evaluación y práctica profesional, pero sufrió la frustración de
\end{abstract}

\footnotetext{
${ }^{1}$ Trabajador Social Investigador, Programa de Trabajo Social, Hospital de Veteranos.

${ }^{2}$ Trabajadora Social, Unidad de Estrés Post-traumático, Hospital de Veteranos.
} 
descubrir que no había sido validado con poblaciones latinoamericanas ni tampoco existía una versión en español autorizada para usos clínicos. Los objetivos de este artículo son: primero, dar a conocer la metodología que se utilizó para adaptar el MPSI al español (Alvelo, 2002) y segundo, presentar un resumen de los resultados de la validez y confiabilidad de las pruebas (Alvelo, Collazo y Rosario, 2001).

\section{Marco Conceptual}

\section{Conceptos Básicos de Medición}

La medición es un mecanismo a través del cual estableciendo reglas le asignamos valores o números a la realidad que nos rodea. Mediante el proceso de medición se ordena, clasifica o cuantifica aquello que nosotros llamamos realidad. En nuestro encuentro con la realidad aspiramos a tener una percepción lo mas objetiva posible. En la medida que la herramienta de medición refleje adecuadamente la realidad que se supone que mida objetivamente así es su grado de validez. Por tanto, una herramienta de medición es válida si cumple con el propósito de medir aquello para lo que fue diseñada. Los métodos para la determinación de validez son los siguientes: apariencia, contenido, criterio y teórica (referirse al Anejo 1 para una definición de las distintas maneras de determinar la validez).

Por otro lado cuando medimos la realidad no solamente aspiramos a tener una representación adecuada de la misma sino que también aspiramos a que sea lo mas precisa y consistente posible en ordenar, clasificar o cuantificar el fenómeno observado. Cuando evaluamos cuán consistente o precisa es una herramienta de medición nos estamos refiriendo a su grado de confiabilidad. Los métodos más comunes de determinar la confiabilidad de un instrumento de medición son los siguientes: prueba-reprueba, formas alternas o paralelas y la división en mitades o consistencia interna (Ander-Egg, 1995). También se utiliza el error estándar de medición y el grado de concordancia entre observadores (referirse al Anejo 1 para una descripción de las distintas maneras de evaluar la confiabilidad).

Hay metodologías cuantitativas y cualitativas para medir la realidad. La metodología cuantitativa está principalmente orientada a generar resúmenes numéricos de aquello observado mientras que 
la cualitativa se orienta a percibir la realidad de forma holística, enfatizando los procesos y la perspectiva de los participantes de esos procesos. Ambas metodologías son igualmente importantes y se complementan. Bloom, Fisher y Orme (2003) mencionan un grupo de métodos de medición que puede utilizar el trabajador social en su práctica profesional, entre ellos están: contar la conducta observada; usar instrumentos de medición estandarizados; utilizar escalas de estimación propia y de otros; el uso de la bitácora por el cliente y medidas no-reactivas (referirse al Anejo 2 para una descripción detallada de algunas de las diferentes metodologías de medición).

El MPSI cae en la categoría de instrumentos estandarizados dentro de los métodos cuantitativos de medición. Para su uso, al igual que para cualquier otra de las metodologías de medición; es importante que el trabajador social tenga unos criterios profesionales para evaluar cuán idóneo es para su práctica profesional.

\section{Ei uso de instrumentos estandarizados}

Lo que distingue a los instrumentos estandarizados de otras técnicas de observación es que tienen procedimientos uniformes para ser administrados. La promoción de los instrumentos estandarizados ha ido en aumento en la literatura de trabajo social al promoverse el uso de herramientas de medición para evidenciar la efectividad de nuestras intervenciones profesionales (Archilla, 2002; Blythe y Tripodi, 1989). Bloom, Fischer y Orme (2003) nos señalan la utilidad de los instrumentos estandarizados en la práctica profesional a saber:

- Facilitan y ayudan en los procesos iniciales de evaluación de clientes, identificando áreas de problema, su severidad o magnitud.

- Ayudan en el proceso de establecer objetivos claros y metas sistemáticas y operacionales.

- El uso de cualquier instrumento ayuda al profesional en el proceso de determinar y especificar claramente el tipo de intervención a usar una vez identifique, y defina claramente el problema. 
- Son de utilidad en la re-evaluación periódica de los planes de tratamiento.

- Nos ayudan también a hacer cambios y ajustes necesarios en los planes de tratamiento iniciales. Vamos a tener información objetiva y científicamente medible.

- Ayudan en la evaluación de clientes que han finalizado tratamiento. Nos ayuda a documentar logros obtenidos de forma objetiva y habiendo sido medida.

- Nos ayudan a integrar la práctica profesional con la investigación científica.

- Pueden ser utilizadas al evaluar programas de tratamiento, su efectividad y su costo.

- Los datos obtenidos pueden también ser usados en la justificación de programas, preparación de propuestas y/o ayudar a una mejor utilización de los recursos disponibles.

Los mismos autores señalan que el trabajador social al evaluar instrumentos estandarizados para ser utilizados en la práctica clínica, investigación u otra tarea de orden administrativa debe definitivamente llevar a cabo una revisión completa del mismo que debe comenzar con leer la información que incluye o debe incluir el manual técnico. Es importante identificar los propósitos del instrumento, lo que pretende medir, su validez y confiabilidad. Debe además, revisar el tipo de investigación científica utilizada para validar el mismo y con qué poblaciones. Tiene que asegurarse de que el instrumento está adaptado culturalmente en este caso a clientes de habla hispana. El trabajador social debe revisar y familiarizarse con la forma de administrarlo, corregirlo (determinar las puntuaciones) e interpretarlo. Hay que revisar cuidadosamente las recomendaciones y observaciones que nos ofrecen los autores de las mismas, incluyendo las fortalezas y limitaciones del instrumento.

Cabe señalar que traducir un instrumento de medición de otro idioma al español no es suficiente para validarlo, por muy buena que sea la traducción (Hernández-Sampieri, Fernández-Collado y 
Baptista- Lucio, 2003, p. 357. De igual forma, Bloom, Fischer y Orme (2003) recomiendan que los trabajadores sociales sean más rigurosos al momento de escoger instrumentos de medición al punto que no tan solo haya evidencia de su validación sino también que sus niveles de confiabilidad excedan el criterio de unos coeficientes de confiabilidad de .80. Los autores apuntan a la necesidad de utilizar una metodología de adaptación transcultural que no sólo incluya la tecnología de su traducción al español sino también las pruebas de psicometría requeridas para la determinación de su confiabilidad y validez.

\section{Modelo de Equivalencia Transcultural}

En el trabajo de adaptación del MPSI para Puerto Rico se utilizó el modelo de equivalencia transcultural propuesto por Bravo, Canino, Rubio-Stipec y Woodbury-Fariña (1991) que se inspiró en el trabajo de Flaherty (1987). En su trabajo con instrumentos de medición para usos clínicos, estos autores utilizaron las técnicas de la metodología transcultural guiados por el modelo de equivalencia cultural. En el mismo, explican el paradigma de la perspectiva emic-etic en la investigación transcultural. La perspectiva emic en la investigación pretende entender el fenómeno bajo estudio desde la cultura misma y en su contexto. Algo similar a lo que haría el antropólogo en sus estudios de culturas distintas a la nuestra. Por otro lado, la perspectiva etic trata de observar el fenómeno desde fuera de la cultura buscando su semejanza con fenómenos equivalentes en otros grupos culturales. En parte, descansa en el supuesto de que la naturaleza humana tenga dimensiones universales a través de las distintas culturas. El modelo de equivalencia cultural incorpora ambas perspectivas. En síntesis este modelo reconoce lo singular de una cultural mientras reconoce la existencia de fenómenos equivalentes a través de culturas diferentes.

Es importante aclarar, que el modelo propuesto por estos autores no descarta el impacto que pueda tener la idiosincrasia cultural, pero tampoco apoya una posición de relativismo cultural absoluto. Para que se pueda trabajar con el modelo es un requisito estar versado en las culturas bajo estudio y esto en muchos casos representa un reto extraordinario en aquellos países que han tenido muy poca exposición a la otra cultura. Pero, no por ser un reto la tarea es imposible y los autores elaboran una serie de estrategias dirigidas a sobreponer este reto. 
En su acercamiento a la adaptación de instrumentos en nuestra cultura Bravo, Canino y Bird (1987) identifican las siguientes cinco dimensiones del modelo para obtener equivalencia transcultural: equivalencia semántica, equivalencia de contenido, equivalencia de técnica, equivalencia de criterio y equivalencia conceptual. La equivalencia semántica examina si el significado de los ítemes es similar entre los lenguajes. La equivalencia de contenido evalúa si el contenido del ítem es pertinente a ambas culturas. La equivalencia técnica juzga si el resultado obtenido con la herramienta utilizada para medir será afectado por las diferencias culturales. La equivalencia de criterio determina si lo medido es interpretado de forma similar conforme a las normas de cada cultura. La equivalencia conceptual pondera si estamos midiendo el mismo concepto en ambas culturas.

Las dimensiones propuestas por este modelo requerirán no solamente una traducción adecuada para obtener equivalencia semántica con relación a la corrección gramatical, nivel del lenguaje y sencillez que faciliten la comprensión del instrumento de medición sino que también hay que construir todo un andamiaje formal de procedimientos que nos asegure hasta donde sea posible que el instrumento desarrollado reúna los criterios de validez y confiabilidad establecidos por la psicometría. A tales efectos los autores en la siguiente sección elaboran las actividades que han de llevarse a cabo para implementar o operacionalizar el modelo de equivalencia transcultural.

\section{El modelo conceptual y su operacionalización}

Las dimensiones del modelo de equivalencia transcultural se operacionalizan mediante el uso de las siguientes técnicas (Bravo, Canino, Rubio-Stipec y Woodbury-Fariña 1991): traducción independiente del documento al español; un comité bilingüe para evaluar los resultados de la traducción y pruebas del mismo; traducción inversa; prueba de instrumento con sujetos; pruebas de campo con grupos conocidos y pruebas de campo para determinar como los conceptos observados se relacionan con otros conceptos.

La traducción independiente se lleva a cabo utilizando los servicios de un traductor profesional certificado con experiencia en la cultura a la que se adapta el instrumento que nos asegure una versión lo mas fiel posible al original pero gramaticalmente correcta y apropiada al país en que se va a utilizar el instrumento de medición 
. La traducción inversa es una técnica transcultural que consiste en tomar la versión ya traducida al lenguaje deseado (español en nuestro caso) y someterla a otro traductor para que la traduzca de nuevo al lenguaje original de la prueba (inglés en nuestro estudio).

El comité bilingüe está compuesto por peritos versados en el campo o disciplina en que se va utilizar la prueba y que a la vez sean versados en los idiomas en que se está adaptando la prueba. Los miembros del comité bilingüe deben haber estado expuestos a ambas culturas y deben tener la capacidad para detectar las idiosincrasias culturales y lingüísticas de cada una. El comité bilingüe revisa y analiza la traducción inicial del instrumento, la traducción inversa y las compara con la versión original. Su misión es asegurarse que la traducción tenga equivalencia semántica, equivalencia de contenido y cultural mediante la evaluación comprensiva de cada ítem con relación a la escala a la que pertenece, el concepto que pretende medir y las idiosincrasias de las culturas comparadas.

La equivalencia técnica, de criterio y conceptual se obtienen mediante las pruebas de campo. Las pruebas de campo son las requeridas por la psicometría en sus distintas vertientes para determinar confiabilidad y validez, a saber: pruebas que determinen la confiabilidad o consistencia interna, pruebas de comprensión, pruebas de validez de contenido, pruebas de validez de criterio y pruebas de validez teórica. Para asegurar que la prueba sea comprensible y culturalmente apropiada se prueba con grupos de sujetos de la cultura a la que se va adaptar. Los sujetos deben poder entender claramente las aseveraciones del instrumento de medición y responder apropiadamente a los mismos.

Entendemos que las pruebas de validez de contenido para una prueba psicosocial requerirá el uso de paneles de expertos en los problemas sociales y sicológicos que comúnmente atienden los clientes de los trabajadores sociales para que determinen la pertinencia de cada ítem al problema que se pretende medir.

Una prueba de validez de criterio es la de validez concurrente que consiste en determinar si la prueba es capaz de discriminar entre poblaciones que tienen el problema de las que no lo tienen.

La relación de conceptos es parte de la validación teórica que se establece basándose en cómo los conceptos se relacionan unos con otros conforme a lo establecido en la teoría.

Durante todas las etapas del proceso es recomendable contar con la asesoría de un perito en el campo de la psicometría. En la 
opinión de los autores, este perito junto al comité bilingüe es fundamental al equipo profesional requerido para implementar el modelo de equivalencia transcultural.

\section{El Inventario para el Cernimiento de Múltiples Problemas}

El Inventario para el Cernimiento de Problemas Múltiples es un instrumento de medición con un enfoque en la evaluación de problemas. El MPSI es una herramienta de evaluación auto-administrable que fue diseñada para medir el grado, severidad, ó magnitud de 27 problemas distintos relacionados con el funcionamiento personal y social. El mismo consiste de 334 aseveraciones. La respuesta a cada aseveración es estandarizada y consiste en escoger la misma de una escala de frecuencia de siete puntos, a saber: 1 = Nunca; 2 = Muy rara vez; 3 = Pocas veces; $4=$ Algunas veces; $5=$ Gran parte del tiempo; 6 = Casi siempre; y 7 = Siempre (referirse al Anejo 3 para una ilustración de una de las escalas). Una vez completados, los 334 ítems están diseñados para producir 27 puntuaciones, una por cada sub-escala que lo compone. Con las puntuaciones obtenidas se prepara un perfil gráfico que facilita su uso en la evaluación y planificación de tratamiento (referise al Anejo 4).

El Inventario requiere de 30 a 45 minutos para completarse. Cada escala tiene una puntuación clínica de corte que le indica al trabajador social la necesidad de intervención o por lo menos la necesidad de evaluar mas exhaustivamente cada problema que exceda el punto clínico de corte, y/o una indicación de resolución o de no tener problema cuando la puntuación no llega al punto clínico de corte. Puede administrarse a poblaciones adultas y a niños mayores de 12 años. Las 27 áreas de problemas sicosociales que evalúa son los problemas más frecuentemente tratados o encontrados por los trabajadores sociales en práctica clínica.

Las 27 escalas del MPSI miden tres dimensiones de problemas funcionamiento psico-social, a saber: funcionamiento individual, relaciones familiares más el entorno social, y relaciones de pareja. El área de problemas de funcionamiento individual incluye las siguientes escalas: depresión, temor, sentimientos de culpa, autoestima, fobias, pensamientos confusos, agresividad, estrés, pensamientos perturbadores, problemas de memoria, pensamientos suicidas, ideas de referencia, abuso de alcohol y drogas. La próxima área que el autor llama la de problemas familiares y entorno social, incluye las 
escalas de problemas con los hijos, con la madre, con el padre, con los amigos, con los vecinos, en la escuela, en el trabajo, con los compañeros de trabajo y las relaciones de familia. Por último el autor agrupa el área de problemas de relaciones de pareja que incluye las siguientes escalas: problemas de pareja, discordia sexual, maltrato físico, y maltrato no físico.

El propósito al desarrollar el MPSI fue proveer una herramienta de evaluación que se utilizaría principalmente durante la admisión o las primeras fases de tratamiento o prestación de servicios a los clientes. Aunque el uso principal del MPSI se enfoca en la evaluación inicial de los problemas, se puede utilizar también para re-evaluaciones periódicas, en la fase final, y de seguimiento posterior al tratamiento. Al utilizarlo de esa manera, proveerá una descripción de cambio (progreso, estabilidad, o deterioro) que pudo haber ocurrido durante el periodo de tiempo entre administraciones. Además de su uso con individuos, se puede utilizar con parejas, con el grupo familiar, grupos focales, en la evaluación de programas, y en la investigación científica. El trabajador social clínico también lo puede utilizar en la auto-evaluación de la efectividad de su intervención.

Hudson (1997) desarrolló el MPSI tras una carrera profesional en trabajo social de mas de 30 años dedicados al desarrollo de pruebas para medir problemas que podrían utilizarse para evaluar la práctica profesional. La teoría que guía la creación del MPSI es una en la que cada escala es el equivalente a un termómetro aplicado a determinar la intensidad de un problema humano. Es necesario mencionar que el MPSI no es un inventario de personalidad, no mide sicopatología o enfermedad mental. En su analogía del termómetro, Hudson aclara que una puntuación alta es indicativa de que hay un problema que requiere ser evaluado para posible intervención pero la misma no nos establece un diagnóstico final ni tampoco establece la causa del problema.

\section{Revisión de Estudios Previos}

La validación inicial del inventario (27 escalas) utilizó una población de 331 estudiantes de bachillerato y de maestría de siete programas de trabajo social en los Estados Unidos de América. En el desarrollo y pruebas de campo de las escalas Hudson (1997) utilizó la teoría clásica de psicometría y aplicó el modelo de muestreo por dominio conforme a la descripción de Nunnally (1978). Los resultados 
de ese primer estudio indicaron que todas las escalas excepto una tenían un índice de consistencia interna medido por el coeficiente alfa que excedían .80 y 16 de ellas excedían .90 (la excepción fue la de agresión con .71). Las pruebas de validez indicaron que las escalas tenían niveles aceptables de validez factorial y de constructo. Solamente las escalas de problemas con los hijos e ideas de referencia obtuvieron fallas factoriales de un $15.4 \%$ y un $5 \%$ respectivamente. La prueba de validez divergente indicó que las escalas en general tenían poca correlación con las variables con las que no se suponían que se relacionaran. Este estudio inicial no tuvo un estudio de validez de contenido.

Posterior al estudio inicial de validación, se completaron dos estudios adicionales donde las propiedades psicométricas de las escalas del MPSI fueron evaluadas. El primero fue una muestra clínica de veteranos del Golfo Pérsico de los Estados Unidos de América, en donde Murphy (Hudson, 1999) encontró que la confiabilidad de las escalas en general fue más alta. Todas, excepto una, excedían el .80 y 21 de ellas el .90 (la de problemas de trabajo obtuvo un coeficiente de .79). El segundo estudio consistió de una batería breve para adultos configurada de un sub-grupo de 16 de las escalas de MPSI por Smith, Arnold, Salston, Heindel y Hudson (Hudson, 1999) en donde todas las escalas excedieron la confiabilidad interna de.80 y nueve de ellas .90. Los estudios revisados sobre las escalas del MPSI no contenían información sobre su aplicación a poblaciones hispanas y Hudson (1999) reconocía la necesidad de continuar evaluando la validez, confiabilidad y las puntuaciones clínicas de corte de las mismas.

\section{Metodología}

El estudio para adaptar a Puerto Rico el MPSI aplicó la metodología transcultural en dos fases: la primera, consistió en la adaptación al español del instrumento y la segunda, fue una prueba de campo que consistió en la evaluación de validez de contenido por un panel de expertos en el área de salud mental seguida por las pruebas de campo para determinar la confiabilidad y validez de las escalas con una población similar a la utilizada por Hudson (1997) en su primer estudio. Es decir, se utilizó en la prueba de campo los estudiantes de bachillerato y de maestría de los programas acreditados de trabajo social de dos universidades de Puerto Rico.

La operacionalización del modelo de equivalencia transcultural 
se ilustra en la figura número 1. Nótese que en la primera fase se generaron cuatro versiones del MPSI. Esto se debió a que después de la traducción inicial, de la traducción inversa y de la prueba con 25 sujetos el comité bilingüe revisaba y hacía ajustes a las mismas. La adaptación al español de un instrumento con derechos de autor presenta unos retos especiales al tratar de mantener la mayor fidelidad posible a la versión original. Los principales retos encontrados fueron los siguientes: 1) abordar rigurosamente el lenguaje coloquial del idioma inglés para adaptarlo al español; 2) no encontrar en el idioma español conceptos o términos idénticos a los del idioma inglés; 3) encontrar expresiones y palabras que se podían traducir literalmente pero que no leían correctamente en el idioma español y 4) hubo un ítem que presentó dificultades por diferencias tecnológicas entre Puerto Rico y los Estados Unidos(el ítem hacía referencia a los trenes como medio de transportación cuando en Puerto Rico no existían tales para la fecha de la prueba del instrumento). Las técnicas para afrontar estos retos con detalles y ejemplos de las mismas fueron descritos en el escrito o publicación por Alvelo (2002).

El comité bilingüe estuvo constituido por tres disciplinas profesionales de la conducta humana, a saber: tres trabajadoras sociales, 2 psicólogos y un siquiatra. Todos con vasta experiencia clínica, credenciales profesionales, experiencia en la investigación y experiencia en la adaptación de documentos del inglés al español. Las tres trabajadoras sociales poseían un grado doctoral, se desempeñaban como catedráticas universitarias, tenían vasta experiencia clínica, experiencia en el campo de la investigación clínica y habían trabajado en diversos proyectos de traducción y adaptación de documentos al español. Los dos psicólogos del grupo tenían una trayectoria profesional en el campo de la psicometría, el grado doctoral, experiencia en la investigación, publicaciones y vasta experiencia en la adaptación de instrumentos al español. El siquiatra era el jefe del departamento de psiquiatría de un escenario médico que prestaba servicios a diversas poblaciones clínicas y con experiencia en las idiosincrasias particulares de las condiciones mentales en la cultura puertorriqueña. Durante los trabajos del comité se hizo un esfuerzo constante para evitar los regionalismos usando como referencia el diccionario de la Real Academia Española y así ofrecer una escala con el potencial de uso en otros países latinoamericanos.

Se hizo una prueba de comprensión de las escalas con 25 sujetos adultos de varias clínicas de salud mental de un hospital de San 
Juan, Puerto Rico. A estos se les pidió que leyesen cada una de las aseveraciones del MPSI y se les preguntó si entendían bien la pregunta. A estos también se les pidió recomendaciones sobre cómo podría expresarse el ítem en una forma más clara y apropiada. Todas las observaciones y recomendaciones sobre esta prueba fueron compiladas por dos asistentes de investigación en una forma estandarizada diseñada para esos propósitos. Tras obtener el consentimiento de los sujetos, cada entrevista fue grabada con el fin de asegurar el tener la información completa, precisa y poder verificar las respuestas si fuese necesario. Las observaciones y recomendaciones fueron posteriormente compiladas y analizadas. El comité bilingüe evaluó cada una de las recomendaciones e hizo cambios en aquellos casos que lo creyó pertinente.

Otro componente de la validación del MPSI fue un estudio de validez de contenido en el cual un panel de expertos independientes juzgó la pertinencia de cada uno de los ítemes de las 27 escalas del MPSI. El panel de 13 profesionales en el área de salud mental estuvo compuesto por psiquiatras, psicólogos, trabajadores sociales y enfermeras siquiátricas. Basados en la cantidad de jueces que juzgaron cada ítem pertinente a su escala se computó un Índice de Validez de Contenido. Un ítem se juzgó pertinente si 11 o más jueces concordaban en la pertinencia del mismo.

En la prueba de campo en Puerto Rico se intentó determinar hasta que punto las propiedades sicométricas de las escalas eran similares al estudio original y así determinar si se podía establecer equivalencia técnica y conceptual. Como estrategia, en la prueba de campo se replicó la metodología del estudio llevado a cabo por Hudson (1997). Además, se hizo un esfuerzo particular en reclutar una muestra lo más similar a la muestra original con miras a controlar, hasta donde fuese posible, variabilidad atribuible a la diferencia de las características de los sujetos.

Para determinar confiabilidad se computó el coeficiente alfa para cada una de las escalas. Para determinar la validez de las escalas se computó la validez factorial y la validez divergente. La validez factorial consistió en explorar si cada ítem de la escala tenía una relación mayor con su propia escala que con las restantes 26 escalas. El cómputo de la correlación del ítem con la escala requiere que se remueva el valor del ítem en el cómputo de la puntuación de la escala para controlar así la correlación del ítem consigo mismo. Este procedimiento generó 27 tablas de correlaciones. Se computó un 
por ciento de fallas cuando en las tablas de correlaciones se identificaban coeficientes mayores a la correlación del ítem con su propia escala. La validez divergente se determinó correlacionando las escalas con variables con las que teóricamente no deberían tener correlación. No habría razón teórica para que las escalas se relacionaran con los datos demográficos de los sujetos (género, estado civil, edad, educación, ingreso, número de veces que contrajo matrimonio, años de matrimonio, número de hijos y tamaño del grupo familiar).

Un resumen de los resultados estadísticos se expone en la próxima sección de este artículo. Los autores sugieren al lector que revise los artículos ya publicados sobre el estudio y al manual técnico del MPSI si desea obtener un análisis comparativo más exhaustivo (Alvelo, Collazo y Rosario, 2001).

\section{Resultados}

Los sujetos del estudio que participaron fueron 313 estudiantes de bachillerato y de maestría pertenecientes a dos universidades de programas de trabajo social acreditados en San Juan, Puerto Rico. Los datos demográficos reflejaron similitud de las muestras de estudiantes de Puerto Rico y de Norteamérica en las áreas de género (mayoría femenina en ambas culturas), años de educación (16.4 y 16.4 ) y estado civil (solteros $60 \%$ y $70 \%$ ). Hubo diferencias en la muestra de Puerto Rico en la edad (6 años menos con un promedio de 26.9), el promedio de ingreso familiar anual en donde los ingresos de las familias de Puerto Rico fue menor ( $\$ 20,500$ versus $\$ 40,000)$ y el tamaño promedio de las familias de Puerto Rico fue el doble al de los norteamericanos (3.4 versus 1.8). Las diferencias de ingreso y tamaño familiar de los sujetos eran de esperarse por las diferencias de ingreso entre Puerto Rico y los Estados Unidos y por las diferencias en el tamaño promedio de las familias en ambas culturas. La diferencia de edad aun mantiene a los grupos en la categoría de adultos jóvenes. A juicio de los autores, lo esencial de estos dos grupos es que ambos son poblaciones no-clínicas de adultos universitarios en dónde no se espera una cantidad substancial de puntuaciones altas en las escalas indicativas de problemas sicosociales.

En el estudio de validez de contenido, el 98 por ciento de los 334 ítemes se consideraron válidos. Solamente 5 ítemes no llenaron los requisitos de validez. Exceptuando estos cinco ítemes se considera que las 27 escalas tienen validez de contenido. 
En general, la confiabilidad de las escalas en español fueron consistentes en sus propiedades psicométricas a las del estudio de Hudson y McMurtry (1997) en términos de magnitud aunque un tanto mas bajas. Dos de las escalas no obtuvieron una confiabilidad de más de .80: la escala de Problemas en el Trabajo obtuvo un coeficiente alfa de .77 y la de Problemas en la Escuela de .68.

Los resultados de validez factorial, validez para discriminar, y validez convergente reflejaron un comportamiento similar al estudio de Hudson y McMurtry revelando que las escalas poseen validez factorial (éxito en el 98.3\%), para discriminar (valor promedio de .08) e indicios de validez convergente al examinar las correlaciones teóricas entre las escalas.

\section{Discusión}

La rigurosidad del método utilizado aplicando el modelo de equivalencia cultural para adaptar el inventario del MPSI al espanol nos mueve a recomendar al el uso experimental de la batería para fines clínicos ya que exhibió altos niveles de confiabilidad y de validez. La misma debe verse como una herramienta para complementar el proceso de diagnóstico social y subordinada siempre al juicio clínico del trabajador social experimentado.

Los autores consideran que se ha logrado un paso inicial al adaptar las escalas del MPSI al español para que otros investigadores las utilicen como base para llevar a cabo estudios adicionales de validación de la misma. Es necesario recalcar que ninguna herramienta de medición es perfecta y aplicable a toda situación. Va a ser necesario hacer estudios de validación de grupos conocidos con cada una de las sub-escalas y de poner a prueba los puntos clínicos de corte.

El autor de las escalas llegó a desarrollar dos baterías más breves especialmente adaptadas para el uso de adolescentes y de adultos. Esto lo hizo utilizando agrupaciones de las sub-escalas del MPSI y la composición de cada cual está descrita en el Manual Técnico del MPSI en su versión en inglés (Hudson, 1999). Consideramos que si la demanda del uso de la batería fuese tal que tuviese aceptación en el mercado latinoamericano se podría negociar con el autor del MPSI la impresión y venta de las escalas en un formato individualizado **. De esta forma, cada escala individual podría utilizarse para la evaluación de la práctica usando los diseños de un solo sistema descritos por Archilla (2002). 


\section{Conclusión}

Al finalizar el estudio los autores expresan que en general la batería refleja una confiabilidad de buena (sobre .80) a excelente (sobre .90) que es aceptable para usos clínicos. También los estudios de validez reflejaron niveles validez de contenido y factorial aceptables. Sin embargo, aun resta validar las puntuaciones clínicas de corte, validar las sub-escalas con grupos conocidos y con otras escalas establecidas.

\section{Bibliografía}

Alvelo, Jaime, (2002). The Multi-Problem Screening Inventory: From English to Spanish. Social Work Today. September, Vol. 2, Num. 18, 16-18.

Alvelo, Jaime., Collazo, Andrés., \& Rosario, David J. (2001) Comprehensive Assessment Tools for Hispanics: Validation of the Multi-Problem Screening Inventory (MPSI) for Puerto Ricans. Research on Social Work Practice, 11 (3), 699-724.

Ander-Egg, Ezequiel. (1995) Técnicas de Investigación Social. 24 Edición. Argentina, Editorial Lumen.

Archilla-Diaz, Sheila. (2002) La medición de efectividad del trabajo social en Puerto Rico. San Juan, Editorial Edil.

Bloom, Martín, Fischer, Joel \& Orme, John G. (2003) Evaluating Practice: Guidelines for the Accountable Professional. Fourth Edition. Boston, Allyn \& Bacon, Pearson Education, Inc.

Blythe, Betty J. y Tripodi, Tony (1989) Measurement in Direct Social Work Practice. California; Sage Publications.

Bravo, Milagros, Canino, Glorisa J. y Bird, Héctor (1987). La traducción y adaptación del esquema de entrevista diagnóstica DIS en Puerto Rico. Acta Psiquiátrica y Psicológica de América Latina, 33, 27-42.

Bravo, Milagros, Canino, Glorisa. J., Rubio-Stipec, Maritza, y Woodbury-Fariña, Michel (1991). A cross-cultural adaptation of a 
psychiatric epidemiological instrument: The Diagnostic Interview Schedule's adaptation in Puerto Rico. Culture, Medicine and Psychiatry, 15, 1-18.

Colón Castillo, Milagros (2000) El uso del pensamiento y del método científico en la práctica profesional de trabajo social. Análisis, 2 (1), 113-118.

Flaherty, Joseph A. (1987). Appropriate and Inappropriate Research methodologies for Hispanic Mental Health. In Moises Gaviria (ed.). Health and Behavior: Research Agenda for Hispanics. Chicago; University of Illinois Press. pp 177-186.

Hernández-Sampieri, Roberto, Fernández Collado, Carlos y Baptista Lucio, Pilar (2003) Metodología de la Investigación. México, McGraw Hill Interamericana.

Hudson, Walter W. (1999) MPSI Technical Manual. Tallahassee, Florida. Walmyr Publishing Co.

Hudson, Walter W. (2002) Manual técnico del MPSI. Adaptación al español por Alvelo, Jaime; Rosario, David y Bruno, Edwin. Tallahassee, Florida. Walmyr Publishing Co.

Hudson, Walter W. y McMurtry, Steven L. (1997). Comprehensive Assessment in Social Work Practice: The Multi-Problem Screening Inventory. Research on Social Work Practice, 7 (1), 79-96.

Nunnally, Jum C. (1978). Psychometric theory. Second Edition. New York: McGraw-Hill.

\section{Notas}

1. La adaptación al español del MPSI fue hecha por Jaime Alvelo, Andrés A. Collazo y David Rosario, con fondos de investigación provenientes de la Administración de Veteranos de los Estados Unidos de América, a través del proyecto de investigación HSR\&D IIR \#94-090.

2. El Manual Técnico del MPSI, el Inventario para el Cernimiento de Múltiples Problemas y el programa para el cómputo de las puntuaciones se obtienen escribiendo a Walmyr Publishing Co., P.O. Box 12217, Tallahassee, Florida, 32317-2217. También se pueden ordenar por Internet en http://www.walmyr.com/ 


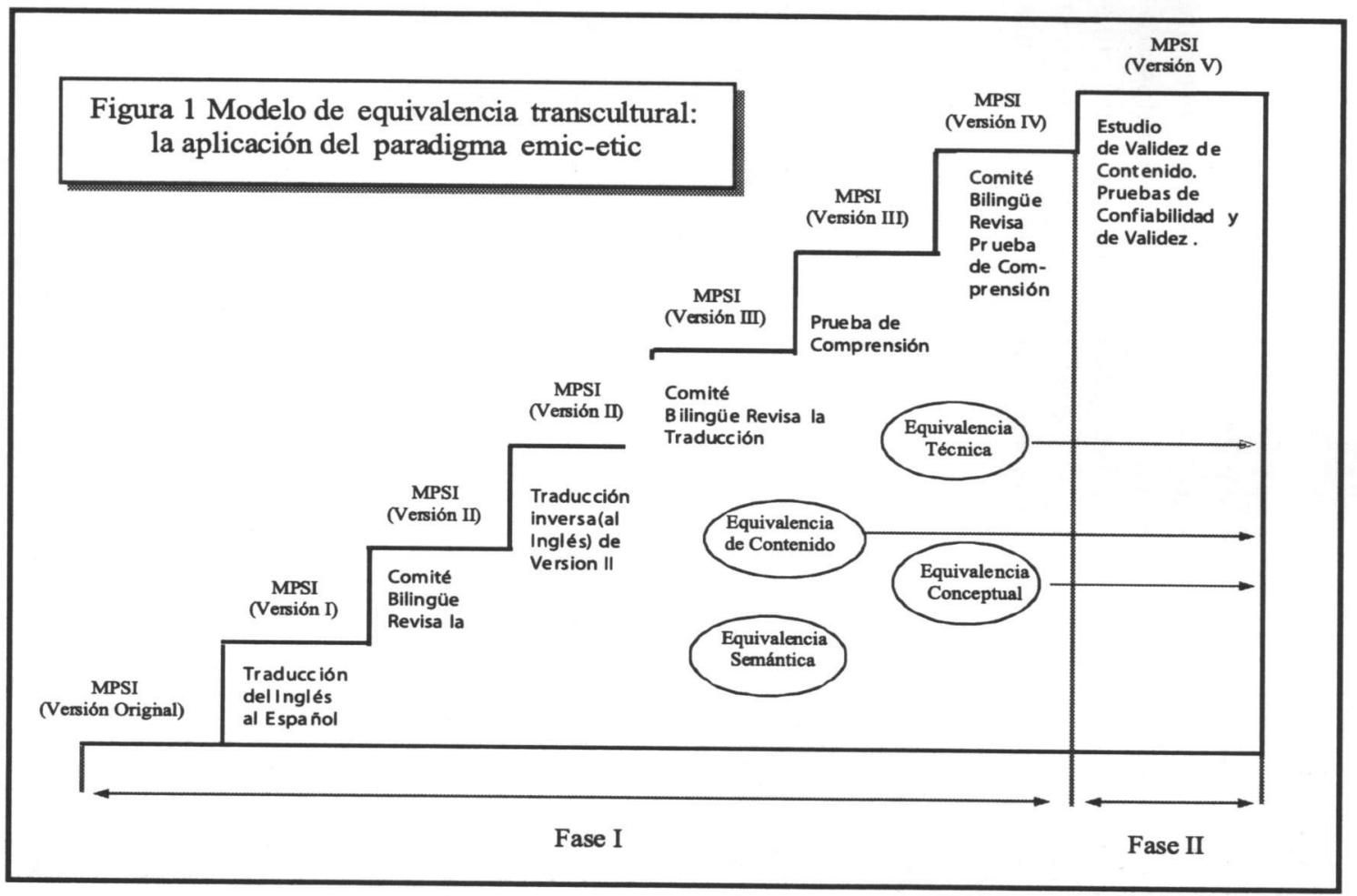

Figura 1. El modelo de equivalencia transcultural utilizado en la adaptación del MPSI al español 


\section{ANEJO 1 \\ Resumen de los conceptos de Confiabilidad y Validez}

\section{CONFIABILIDAD}

a) Prueba-reprueba (Test-retest): Esta prueba determina la estabilidad del instrumento a través del tiempo. Básicamente consiste en dar la misma prueba a los mismos sujetos en dos ocasiones diferentes. Será confiable si las puntuaciones no fluctúan demasiado y los sujetos se mantienen relativamente en la misma jerarquía. Debe indicarse el intervalo de tiempo entre las pruebas.

b) División a Medias o por Mitades (Split-half reliability): Determina la consistencia interna de un instrumento. Consiste en dividir todos los ítemes que mide una variable en dos partes iguales (e.g. si hay 50, en dos grupos de 25) y ver como estos se relacionan unos con otros. Será confiable el instrumento si ordena a los sujetos en una forma consistente.(La relación entre ambas partes se le conoce como el coeficiente alfa.)

c) De formas alternas (Alternate forms): La confiabilidad se determina midiendo la variable con otro instrumento diseñado para medir lo mismo. La medición se lleva a cabo en tiempos diferentes. Se recomienda su uso para evitar el problema de que los sujetos puedan memorizar los reactivos y así inflar el índice de confiabilidad.

d) Error estándar de medición (Standard Error of MeasurementSEM): Consiste en determinar cual es el margen de error de la puntuación de una prueba. Entre mas pequeño es este número más confiable es su prueba ya que menor es el margen de error.

e) Concordancia entre observadores (Interrater reliability): Se refiere a cuan consistente son dos o mas observadores al clasificar un mismo fenómeno. Se expresa como un por ciento de concordancia. 


\section{VALIDEZ}

1- De Apariencia (Face): Se juzga un instrumento de medición valido en base a nuestra impresión subjetiva de cuan razonables son las preguntas al intentar medir la variable bajo estudio.

2- De Contenido (Content): La validez de contenido se juzga en base a cuan representativo son las preguntas del concepto que se pretende medir.

Ejemplo: Si pretendo medir habilidad matemática. Mi examen tendrá validez de contenido si cubre adecuadamente los componentes del concepto de habilidad matemática: problemas de suma, resta, multiplicación y división.

3- De Criterio (Criterion): La validez del instrumento de medición se juzga comparando los resultados de este con otros criterios ya establecidos que miden la variable bajo estudio.Existen dos categorías:

a) Validez Concurrente (Concurrent): La habilidad de un instrumento de medición para distinguir entre grupos conocidos.

b) Validez Predictiva (Predictive): La habilidad de un instrumento para predecir funcionamiento futuro basado en funcionamiento actual.

4- Validez de Constructo o Teórica (Construct): La validez del instrumento de medición se establece sobre la base de cómo los conceptos medidos se relacionan unos con otros conforme a la teoría o el marco conceptual.

\section{ANEJO 2 \\ Metodos de Medición}

\section{LA CONDUCTA}

Definición: Se define como cualquier movimiento, tarea o activiobtener un total de las mismas. Cuando se cuenta se puede indicar cuán a menudo ocurre un problema (frecuencia), cuánto tiempo dura el mismo (duración). También se puede obtener cuantas veces ocurre un problema en un período de tiempo (intervalo). 


\section{Instrumentos De Medición Estandarizados}

Definición: Son los instrumentos que tienen procedimientos uniformes para administrarse. Un instrumento estandarizado tendrá información escrita sobre lo que pretende medir; instrucciones la forma de administrarse; los pasos para determinar la puntuación de los sujetos; una explicación de como interpretar las puntuaciones; los parámetros o las normas de la población en que se desarrolló; estimados sobre su confiabilidad y los procedimientos utilizados para validarlo.

\section{Escalas De Estimación: Propia y Por Otros}

Escalas de Estimación Propia (Self-Anchored rating Scale): Es un instrumento de medición redactado de tal forma que el mismo sujeto se sitúa en algún punto de una serie ordenada de categorías de una variable uni-dimensional.

Escala de Estimación por Otros (Rating Scale): Es un instrumento de medición redactado de tal forma que un observador sitúa a otra persona en algún punto de una serie ordenada de categorías de una variable uni-dimensional.

\section{La Bitácora del Cliente}

Definición: La Bitácora son notas organizadas llevadas a cabo por el cliente de eventos o incidentes que considera relevantes a su problema, enfocándose en su percepción de los eventos, las situaciones o las inter-acciones alrededor del mismo. El uso de la Bitácora es un intento de ayudar al cliente a que lleve apuntes de los eventos relacionados con su problema en forma objetiva y sistemática para evitar la distorsión de los mismos debidos a fallos de la memoria cuando éste se reune con el TS. Es una manera de obtener insumo sobre le problema y sobre las circunstancias que lo rodean.

\section{La Reactividad y Medidas No-Reactivas}

Reactividad: Esto consiste en cambios en la conducta o en el problema del cliente debido a la medición y no a cambios como resultados de la intervención en sí.

Instrumentos de observación "no-reactivas": Estos son instrumentos de medición en que el cliente no está consciente de que está siendo estudiado o evaluado de tal forma que no se genera reacciones o comportamientos que puedan afectar los datos. 


\section{Métodos Cualitativos en la Ponderación}

Definición: Método de ponderación que le da énfasis al contexto y al proceso mediante la compilación detallada de eventos cuya descripción, significado e interpretación la provee el cliente.

El método depende mas en palabras, retratos, diagramas y la narrativa que en números. Está orientado a obtener información sobre la parte subjetiva de la experiencia humana. Trata de capturar la dinámica de los procesos. El esfuerzo del trabajador social está orientado a tratar de entender el mundo desde el punto de vista del cliente.

\section{ANEJO 3 \\ Ejemplo de una de las escalas del MPSI}

Este cuestionario ha sido diseñado con el propósito de obtener información acerca de una amplia variedad de posibles áreas problemáticas. Responda a cada pregunta con la mayor atención y precisión posible. Coloque un número junto a cada pregunta, como se indica a continuación:

$$
\begin{aligned}
& \text { CLAVE } \\
& 1=\text { Nunca } \\
& 2=\text { Muy rara vez } \\
& 3=\text { Pocas veces } \\
& 4=\text { Algunas veces } \\
& 5=\text { Gran parte del tiempo } \\
& 6=\text { Casi siempre } \\
& 7=\text { Siempre } \\
& \mathrm{x}=\text { No aplica }
\end{aligned}
$$

Puede ser que algunas de las preguntas no se apliquen a usted o a su situación en particular. En tal caso, favor de colocar una $\mathrm{x}$ o X pero NO DEJE RESPUESTA ALGUNA EN BLANCO.

Una vez comience a completar las preguntas en el cuestionario, verá cuán fácil es hacerse pasar como una persona buena o mala. Por favor, no lo haga. Es extremadamente importante que usted conteste con la mayor precisión aunque se avergüence o se sienta incómodo/a. Si responde incorrectamente u ofrece información engañosa a aquellos que tratan de ayudarle, será muy difícil prestarle la ayuda que usted necesita. 


\section{LAS RELACIONES FAMILIARES}

158.

En mi familia hay demasiado odio.

159 .

Los miembros de mi familia se tratan muy bien entre sí. *

160

Mi familia es muy respetada por aquellos que nos conocen. *

161. En mi familia parece haber mucha fricción.

162. En mi familia hay mucho amor. *

163. Los miembros de mi familia se llevan bien entre sí. *

164. Mi vida familiar en general es desagradable.

165 . Mi familia me llena de gran alegría. *

166 . Me siento orgulloso/a de mi familia. *

167. Las demás familias parecen llevarse mejor que la mía.

168 . Mi familia es una fuente verdadera de apoyo. *

169 Siento que mi familia me hecha a un lado (me sacan el cuerpo).

170. Mi familia es infeliz.

* Indica aseveración a ser invertida al computar puntuación

0Copia para fines didácticos de la sub-escala de Problemas en Relaciones Familiares de 27 que componen el MPSI. Se prohíbe su reproducción. Copias pueden ser obtenidas mediante compra de la batería en: WALMYR Publishing Co., P.O. Box 12217, Tallahassee, FL 32317-12217. La batería de 27 escalas del MPSI se puede ordenar por Internet en http://www.walmyr.com/

NOTA SOBRE DERECHOS DE PROPIEDAD (COPYRIGHT): No se permite reproducir ni fotocopiar ninguna parte del Inventario para el Escrutinio de Problemas Múltiples (MPSI por sus siglas en inglés) sin el consentimiento por escrito de la editorial. Copyright (c) 1990, Walter W. Hudson, Ph. D. 


\section{Perfil Gráfico de las Puntuaciones de un Cliente}

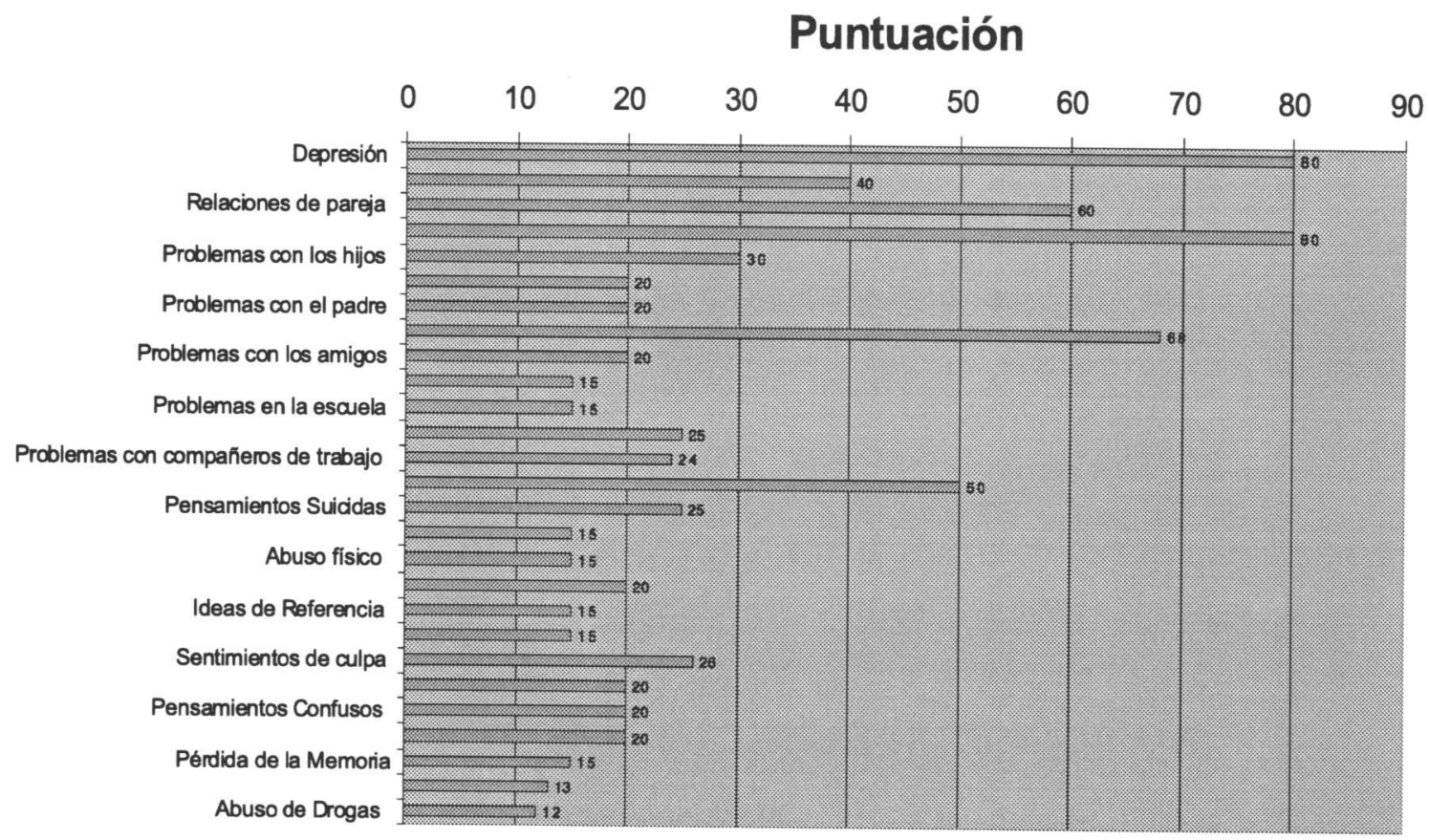

